\title{
OPTOELECTRONIC AND SENSING APPLICATIONS OF PLASMONIC SILVER NANOPARTICLES
}

\author{
Isha Saini $^{1}$, Himanshi ${ }^{2}$, Meena Yadav ${ }^{2}$ \\ *E-mail Id:1'ishasaini@ansaluniversity.edu.in, ${ }^{2}$ himanshigupta89@gmail.com, \\ 2ymeena12@gmail.com \\ School of Engineering and Technology, Ansal University, Gurgaon, Haryana, India
}

\begin{abstract}
In recent time, nanoparticles of noble metals like gold, silver have drawn a considerable interest of researchers due to their various applications such as in sensors, electronic devices, solar cell, semiconductors, biosensors etc. In the present review article, the main focus is on silver nanoparticles (Ag NPs) which are widely used due to their unique properties such as optical, electronic, catalytic properties which are shape \& size dependent. The interaction of light with silver nanoparticles can give rise to collective oscillations of the free electrons commonly known as surface plasmons. The surface plasmon resonance band (SPR) for silver nanoparticles occurs in the visible region of electromagnetic spectrum and this property has been exploited in a number of applications. This paper highlights the various optoelectronic and sensing application of silver nanoparticles.
\end{abstract}

Keywords: Silver nanoparticles, Surface Plasmon Resonance, Optoelectronic properties, Sensors.

\section{INTRODUCTION}

In the past few decades, the world has seen a tremendous growth in the application of nanoscience and nanotechnology, largely due to the special properties that the nanomaterials possess. This is primarily due to their small size and, thusthe exceptional surface area of these materials [1] [2]. In a bulk material, the properties of the material are independent of size and are dependent only on chemical composition. As the size of the material is reduced to the nanometer range, the electronic structure is altered from the continuous electronic bands to discrete or quantized electronic levels. As a result, the continuous optical transition between the electronic bands becomes discrete and the properties of the nanomaterials become size dependent. Nanoparticles of noble metals like gold, silver, platinum and palladium are used in many fields. Ag-NPs, in particular, has attracted researchers due to its unique properties like high thermal and electrical conductivity, surface-enhanced Raman scattering, chemical stability, catalytic activity, and nonlinear optical behavior [3]. One other reason thatAg NPs are gaining considerable attention is also due to their significant surface plasmon resonance (SPR) property. The surface plasmon resonance (SPR) is the coherent motion of the conduction band electrons caused by interaction with an electromagnetic field [4]. This fascinating optical response of silver nanoparticles has been attracting continuous interest of scientist and technologists since long. Due to this extraordinary optical feature, these materials have large number of applications in photonics, sensors, colour filters etc.

The size and geometry of Ag-NPs are highly dependent on the method of its synthesis. Numerous methods of synthesis of Ag NPs have been developed due to its intense use. These methods include physical, chemical and biological methods of synthesis. Physical methods are evaporation/condensation, laser ablation and thermal decomposition. Also silver salt can be reduced to silver nanoparticles using chemical and biological reducing agents. Chemical methods are most widely used due to their simplicity and cost effectiveness. Experimental conditions like $\mathrm{pH}$, temperature, reactants and nature of stabilizers are important factors as they control the size, shape, stability, morphology and color of the nanomaterials [5] [6].

Silver nanoparticles are used in LED, Display devices, catalyst and sensors [4]. Numerous gas sensors have been fabricated to sense the gases like hydrogen, ammonia, oxygen and hydrogen peroxide. In these sensors, silver nanoparticles in the form of nanoparticles or nanocomposites have been extensively used with other materials. Ag NPs enhances the properties of sensors such as response time, selectivity and sensitivity [7]. In this review, we emphasizethe applications of silver nanoparticles in LED, optoelectronics and sensing devices.

\section{SILVER NANOPARTICLES FOR HYDROGEN GAS SENSING}

Hydrogen $\left(\mathrm{H}_{2}\right)$ gas sensors have been used in many areas like chemical plants, energy storage and fuel cells. As hydrogen has no taste, smell and color, it cannot be sensed by human sense. Hence more sensitive, cost effective and reliable hydrogen sensors are being made. Silver nanoparticles due to its extraordinary properties like larger conductivity and more cost efficient as compared to other noble metals like gold, platinum and palladium, is used in gas sensing devices excessively. It can be used as nanocomposite for $\mathrm{H}_{2}$ gas sensing. V.S.Rizi et al.have fabricated sol-gel derived $\mathrm{SnO}_{2} / \mathrm{Ag}_{2} \mathrm{O}$ ceramic nanocomposite which is used for sensing of $\mathrm{H}_{2}$ gas.. The size of CNP was from 20 to $90 \mathrm{~nm}$. Also, uniform distribution of silver in $\mathrm{SnO}_{2}$ matrix was obtained at $300^{\circ} \mathrm{C}$. It was observed that best response of sensor was given by $\mathrm{CNP}$ annealed at $300^{\circ} \mathrm{C}$. This is due to highest uniform distribution of silver in $\mathrm{SnO}_{2}$ matrix at this temperature. Hence, it was revealed that homogeneous distribution of silver nanoparticles in tin oxide makes the sensor more responsive, sensitive and selective [7] [8] [9].

\section{SILVER NANOPARTICLES FOR SENSING OF HYDROGEN PEROXIDE}

Hydrogen peroxide has a wide range of application in industries and medical field for clinical diagnosis [10]. But, large concentration of this analyte affects the human health. Spectrophotometry, chemiluminescence and DOI Number: https://doi.org/10.30780/specialissue-ICACCG2020/029 
International Journal of Technical Research \& Science (Special Issue) ISSN No.:2454-2024 (online) electrochemistry are some of the techniques used to detect hydrogen peroxide. Out of these, electrochemical technique is simple, low cost, easy to operate andhas high sensitivity and selectivity. Metals, metal oxides and organolanthanide complexes are used in electrochemical sensors to overcome some drawbacks like high overpotential. Since, silver nanoparticles have high electron transfer rates and less cost, they are most widely used in these sensors. Catalytic action of silver nanoparticlesfor reduction of $\mathrm{H}_{2} \mathrm{O}_{2}$ and great decrease in overpotential at oxidizing and reducing agents due to silver nanoparticles, make them to be used as main component in fabrication of such kind of sensors. H.Wang et al. have shown that silver nanoparticles are used in graphenecolloidal carbon sphere (Gr-CCS) composites for sensing of hydrogen peroxide $\left(\mathrm{H}_{2} \mathrm{O}_{2}\right)$. In this study, tailored structure carbon based composites with metal nanoparticle doping is synthesized and used to fabricate electrochemical sensors for $\mathrm{H}_{2} \mathrm{O}_{2}$ sensing. AgNPs doped Gr-CCS composites are prepared based on catalytic action of $\mathrm{Ag}$ NPs for detection of $\mathrm{H}_{2} \mathrm{O}_{2}$. Gr-CCS composites were prepared under hydrothermal conditions at $180^{\circ} \mathrm{C}$ and $\mathrm{AgNO}_{3}$ with sodium citrate was used to produce Ag NPs. Ag NPs were deposited onto CCS of GrCCS by electrostatic interaction. Due to good catalytic action of Ag NPs for $\mathrm{H}_{2} \mathrm{O}_{2}$, the Gr-CCS-AgNPs modified GC electrode based sensor was fabricated which show fast response, good selectivity, good sensitivity and good resistance to interference [11] [12].

\section{APPLICATIONS OF SILVER NANOPARTICLES IN ELECTRONICS}

Silver nanoparticles have vast application in microelectronics [13] [14]. When size of nanoparticles reduces, surfaces energy becomes very significant role, same way when AgNPs size decreases its melting point also decrease drastically. Chen et al studied that silver flakes or particles used for filling gaps which arises in the process of preparation of electrically conductive adhesives(ECA). After combination of AgNPs, ECA conductivity improve highly when it preserves at low temperature. In this way AgNPs used as conductive filler in many ECA, which is very useful for various electronics purposes such as photovoltaic charge collection tapes, electromagnetic interface, bonding between electronic components and also in shielding electronic devices [15] [16].

\section{APPLICATION OF SILVER NANOPARTICLES IN SOLAR CELL}

Silver Nanoparticles are also used for storage purpose. Efficiency of solar cells made up of Silicon and CdTe are limited, when designed by conventional methods because of poor absorption and low photo generation .Hence silicon solar cell surface coated with silver Nanoparticles which increases photon injection process,so that absorption of silicon solar cell increases[17]. It is also found that if a layer of silver nanoparticles designedwith silicon then its effectiveness increases because this combined layer increases the refractive index of silicon [18] [19].

\section{APPLICATIONS OF SILVER NANOPARTICLES IN LIGHT EMITTING DIODES}

Silver nanoparticles have different applications for organic and inorganic light emitting diodes because of its special properties of surface Plasmon effect[20].The SPR is a dipolar excitation of the entire particles between negative charged free electron and positive charge lattice.It was found that luminous efficiency of the fluorescent molecules is improved,when fluorescentmolecules come close to the AgNPs.Distance between fluorescent molecules and AgNPs surface play an important role in coupling between excitons and surface Plasmon.Chen et al experimental show thatAgNPs increases the efficiency of OLEDs, when a layer of silver nanoparticles is designed between electrodes and electron transport layer. When a layer of silver nanoparticle designed between electron transport layer ,then efficiency of OLEDs is increased 2.4 times. When the distance between silver Nanoparticles and fluorescent molecules decreases which means they have come closer and thenspontaneous recombination of exciton and electron takes place.In this way efficiency of OLEDs improves[22].Cho et al. showed that the silver nanoparticles increased the efficiency of surface enhanced blue LED. When AgNPs attached with p-GaN layer in SP-enhanced blue LED its efficiency increased 72\% [23].

\section{SURFACE ENHANCED RAMAN SCATTERING}

The surface enhanced raman scattering (SERS) technique is a powerful analytical tool in the fields of surface science, biology, analytical chemistry, biochemistry, catalysis and materials research. The excellent sensitivity and selectivity of SERS allow for the determination of chemical information from single monolayer on planar surfaces and extend the possibilities of surface vibrational spectroscopy to solve a wide array of problems. Silver nanoparticles exhibit surface enhanced raman scattering and are excellent materials for SERSmeasurements because of the broad plasmon resonance band in the visible region, high stability, highsurface enhancements and ease in fabrication.The aggregation of silver nanoparticles is prerequisite for stronger SERS enhancement.Meyer et al [24] has done a systematic study to correlate surface enhancements from aggregated Ag NPs with the mode of interaction between the analyte and metalsurface, analyte structure, excitation wavelength, size of thenanoparticle aggregates, as well as, the stability of the Raman measurements over time.

\section{CONCLUSION}

The growth of the interest on silver nanoparticles is motivated by the rise of various potential applications in different fields of science and technology and are particularly promising candidates for application in the fields of nonlinear integrated optics and photonic devices. Silver Nanoparticles have diverse applications in numerous fields. They have attracted researchers very much from last few decades as they display surface plasmon resonance in the visible region besides their high conductivity and easy availability. In the present review article an attempt has been made to discuss some of the applications of silver in optical devices, energy storage devices and sensing 
International Journal of Technical Research \& Science (Special Issue) ISSN No.:2454-2024 (online) devices. Research is still undergoing to explore the use of silver nanoparticles in energy storage devices to improve their performance.

\section{REFERENCES}

[1] A. V. Nikam, B. L. V. Prasad, and A. A. Kulkarni, "Wet Chemical synthesis of metal oxide nanoparticles: a review", Cryst Eng Comm,Vol. 20, pp 5091-5107 (2018).

[2] A. A. Yaqoob, K. Umar, M. N. M. Ibrahim, "Silver nanoparticles: Various methods of synthesis, size affecting factors and their applications-a review",Appl Nanosci,Vol.10, pp1369-1378 (2020).

[3] P. Dolez," Nanomaterials Definitions,Classification and Application", Nanoengineering: Global approaches to health and safety issues, pp 3-14 (2015).

[4] Dinesh C. Agrawal, Introduction to Nanoscience and Nanomaterials, World Scientific Publishing Co. Pte. Ltd., pp 141-156, (2013).

[5] M. Kumar, P. Devi, and A. Kumar,"Structural analysis of PVP capped silver nanoparticles synthesized at room temperature for optical, electricalandgas sensing properties", J. Mater. Sci. Mater. Electron., Vol. 28, 5014-5020, (2017).

[6] X. F. Zhang, Z. G. Liu, W. Shen, and S. Gurunathan,"Silver nanoparticles:Synthesis,characterization,properties,applicationsand therapeutic approches", Int. J. Mol. Sci.,Vol.17, (2016).

[7] A. Haider and I. Kang,"Preparation of silver nanoparticles and their industrial and biomedical applications: a comprehensive review", Advances in materials science and engineering, Vol. 2015, pp 1-16, (2015).

[8] A. Jeevika and D. R. Shankaran,"Functionalized silver nanoparticle probe for visual calorimetric sensing of mercury", Mater. Res. Bull., Vol. 83, pp 48-55, (2016).

[9] V. S. Rizi, F. Sharifianjazi, H. J. Khorami, N. Parvin,L.S. Fard, M. Irani,A. Esmaeilkhanian,"Sol-gel derived $\mathrm{SnO}_{2} / \mathrm{Ag}_{2} \mathrm{O}$ ceramic nanocompositefor $\mathrm{H}_{2}$ gas sensing applications", Mater. Res. Express, Vol.6, pp 1150g2, (2019).

[10] A.K.M.Khair, Q.A.Drmosh and Z.H.Yamini"Silver nanoparticle decorated tin oxide thin films: Synthesis, characterization and hydrogen gas sensing", Frontiers in materials, Vol. 6, pp 188, (2019).

[11] H. Wang, H. Wang, T. Li, J. Ma, K. Li, and X. Zuo,"Silver nanoparticles selectively deposited on graphenecolloidal carbon sphere composits and their application for hydrogen peroxide sensing", Sensors Actuators, B Chem.,Vol. 239, pp 1205-1212 (2017).

[12] M. T. Ramesan and V. Santhi,"In situ synthesis, characterization,conductivity studies of polypyrrole/silver doped zinc oxide nanocomposits and their application for ammonia gas sensing" J. Mater. Sci. Mater. Electron. Vol.28, pp 18804-18814 (2017).

[13] S. L. C. Hsu and R. T. Wu," Synthesis of contamination free silver nanoparticle suspension for microinterconnects". journal of Material. Letter. 61, 3719 (2007).

[14] C. Y. Cho, K. S. Kim, S. J. Lee, M. K. Kwon, H. Ko, S. T. Kim, G. Y. Jung, and S. J. Park, "Surface plasmonenhanced LED with silver nanoparticles \& $\mathrm{SiO}_{2}$ nano disks embedded in p-GaN Appl. Phys. Lett.vol 99, 2012 (2011).

[15] Pothukuchi S, Li Y, Wong CP "Development of a novel polymer metal nanocomposite obtained through the route of in situ reduction for integral capacitor application". journal of applied polymer,Vol 93 pp 1531$1538,(2004)$.

[16] D.Chen,X.Qiao," Synthesis and electrical properties of uniform silver nanoparticles for electronic application", journal of material science (2009).

[17] J. Winans,J.Clorkson,P.fanchet, "silver nanoparticles to enhance the efficiency of thin film of solar cell". International journal of engineering science, Vol. 2, pp 101-104 (2011).

[18] S.Maity, Sahadev Roy, Abhishek kumar, AgNps to enhance the efficiency of silicon solar cell (2013).

[19] V.Thamilselvi and K.V Radha," A review on the diverse application of silver nanoparticles " IOSR journal of pharmacy, Vol. 7 pp 21-2 (2017).

[20] M.O Alam and C.Bailey,A volume in woodhead Publishing Series in eletronic and optical materials (2011).

[21] [21] G. Cao, "Nanostructures and Nanomaterials synthesis, properties \&application". Imperial College Press, pp 362-36, (2003).

[22] Y. C. Chen, C. Y. Gao, K. L. Chen, T. H. Meen, and C. J. Huang, "Enhancement \&quenching of fluorescence by silver nanoparticles in organic light emitting diodes".journal of Nanomaterials, Research article, Vol. 2013, (2013).

[23] C.Cho,K. Kim,S.Lee," Surface plasmon-enhanced light emitting diodes with silver nanoparticles \& $\mathrm{SiO}_{2}$ nano-disk embedded in p-GaN". Appl Phy Lett, Vol. 99 (2011).

[24] M. W. Meyer and E. A. Smith, Analyst "Optimization of silver nanoparticles for surface enhanced Raman spectroscopy of structurally diverse analytes using visible and near-infrared excitation".journal of Analyst.136 (2011) 3542. 